Cent ans après sa découverte, la supraconductivité fascine toujours. Ceci est lié au fait que ce phénomène se traduit par des comportements insolites à l'échelle macroscopique, comme la disparition de

la résistance électrique ou l'expulsion du champ électromagnétique. Ces effets sont le reflet de la mécanique quantique à notre échelle de perception. Certaines conséquences, comme la circulation de courants électriques permanents ou la quantification du flux magnétique, sont à la base d'applications spécifiques, surtout dans le domaine médical.

En rappelant l'origine de ces phénomènes et les concepts fondamentaux de la supraconductivité, cet article se veut une introduction sur laquelle s'appuiera la suite de ce dossier.

\title{
Supraconducteurs La mécanique quantique à grande échelle
}

Kees van der Beek (kees.vanderbeek@polytechnique.edu)

Laboratoire des Solides Irradiés, UMR CNRS 7642, École polytechnique, 91128 Palaiseau Cedex

La supraconductivité d'un matériau se manifeste par la disparition de toute résistance électrique en dessous d'une température $T_{c}$, appelée température critique. C'est ainsi qu'en 1911 Heike Kamerlingh Onnes et son équipe ont découvert ce phénomène dans le mercure, lorsqu'ils le refroidirent en dessous de 4,20 K [1,2]. Cette découverte fut rapidement suivie d'observations analogues dans l'étain $\left(T_{\mathrm{c}}=3,7 \mathrm{~K}\right)$ et le plomb $\left(T_{\mathrm{c}}=7,2 \mathrm{~K}\right)[1]$.

La transition à $T_{\mathrm{C}}$ traduit un changement d'état au sens thermodynamique. Willem Keesom et J.A. Kok montrèrent en 1932 qu'à $T_{c}$, la chaleur spécifique d'un supraconducteur est discontinue, sans chaleur latente (fig. 1a). En absence d'un champ magnétique, la transition est donc du second ordre [3]. En dessous de $T_{c}$, l'induction magnétique $B$ est nulle au sein du matériau (effet Meissner, fig. 1b).
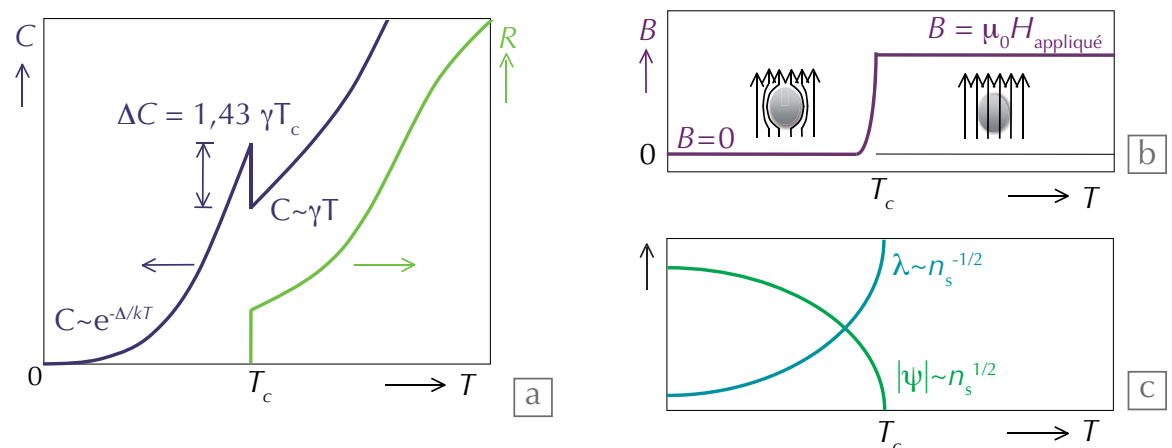

1. Propriétés phénoménologiques d'un supraconducteur.

(a) À la température critique $T_{\text {c }}$, la résistance électrique $R$ chute à 0 . La chaleur spécifique $C$ présente un saut qui, selon la théorie BCS [4], est égal à 1,43 $\gamma T_{c}$, avec $\gamma T$ la contribution des électrons à la chaleur spécifique à l'état normal. À basse température, C suit un comportement exponentiel, ce qui montre l'existence d'une bande interdite d'énergie $\Delta$. (b) En dessous de $T_{c}$, les champs électromagnétiques sont expulsés, de manière à ce que l'induction magnétique $B$ au sein du supraconducteur soit nulle (effet Meissner).

(c) Évolution en température de la norme $|\psi|$ du paramètre d'ordre de Ginzburg et Landau, et de la longueur de pénétration $\lambda .|\psi|^{2}$ et $\lambda^{2}$ sont respectivement proportionnel et inversement proportionnel à la densité d'électrons supraconducteurs, $n_{s}$. 


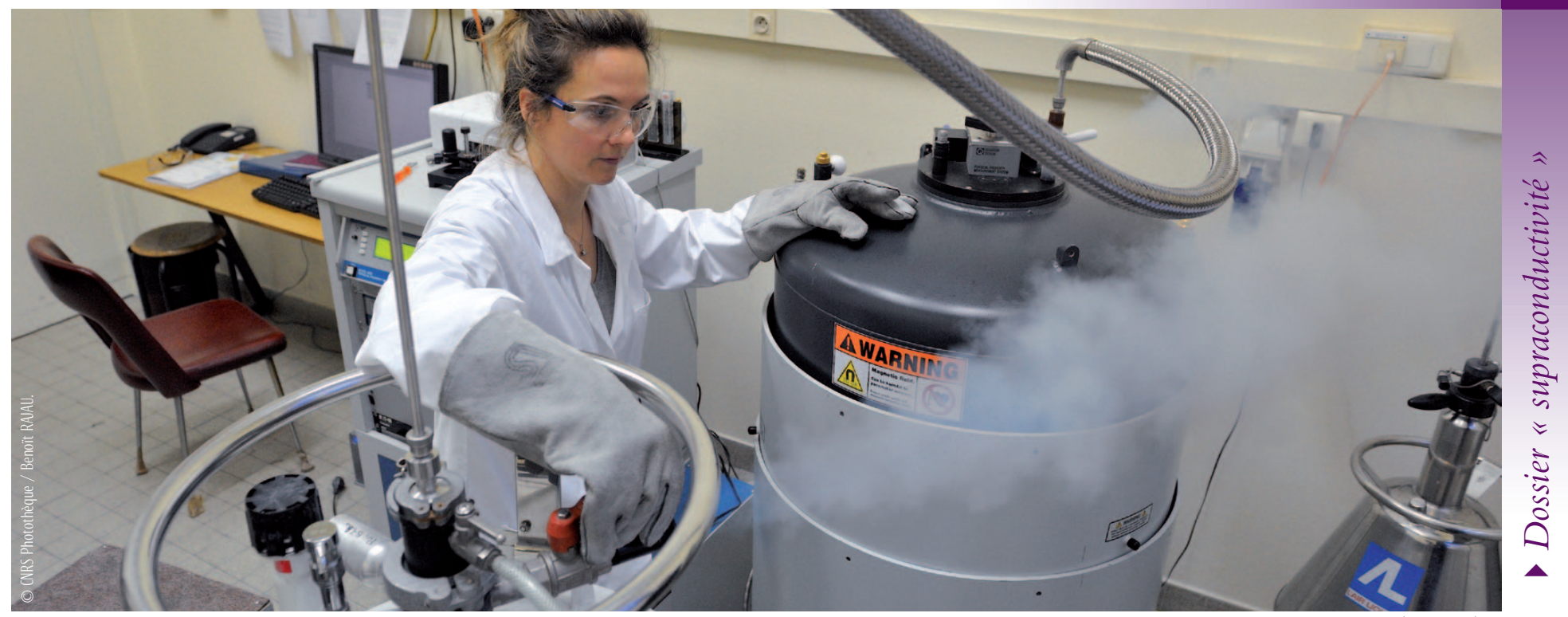

Transfert d'hélium liquide dans un cryostat pour faire des mesures de la résistivité électrique de supraconducteurs, à basse température et sous fort champ critique (14 teslas).

mercure ou le plomb), la supraconductivité disparaît lors d'une transition de premier ordre au champ critique, $H_{c}$, dont la valeur est plutôt modeste $(\sim 80,3 \mathrm{mT}$ pour le plomb). Si, en dessous de $H_{\mathrm{c}}(T)$, le champ magnétique est expulsé, il est de nouveau admis lors du retour du matériau à l'état normal à $H_{c}$. En fait, la supraconductivité disparaît lorsque le coût énergétique associé à l'exclusion du champ (proportionnel au carré de $H$ ), ne peut plus être compensé par la différence en énergie entre l'état normal et l'état supraconducteur en champ nul (fig. 2).

\section{Supraconductivité de type II}

Cependant, la grande majorité des supraconducteurs est de type II. Dans ces matériaux, le champ magnétique est partiellement réadmis au-dessus d'un premier champ critique $H_{\mathrm{c} 1}$. Le champ pénètre alors dans le matériau sous la forme de "vortex ", lignes de flux magnétique quantifié entourées de tourbillons de courant non dissipatif [5]. Les vortex remplissent progressivement le matériau, jusqu'à ce que celui-ci transite vers l'état normal par une transition de second ordre à un deuxième champ critique $H_{\mathrm{c} 2}$. Les premiers supraconducteurs de type II découverts furent des alliages, tel que le $\mathrm{Pb}-\mathrm{In}$. Les champs critiques $H_{\mathrm{c} 1}$ et $H_{\mathrm{c} 2}$ dépendent très sensiblement des propriétés intrinsèques et de la pureté du matériau. Ceci est primordial pour les applications de la supraconductivité, qui sont pour une grande partie tributaires de la valeur de $H_{\mathrm{c} 2}$. Les très grandes valeurs de $H_{\mathrm{c} 2}(\grave{a} T=0)$ des alliages et composés intermétalliques à base de niobium (voir tableau) ont, dans les années 1960, permis la mise au point des électroaimants puissants imaginés par Kamerlingh Onnes. Ces valeurs sont \〉
Températures critiques $T_{\mathrm{c}}$ et champs critiques supérieurs $H_{\mathrm{c} 2}$ de quelques supraconducteurs importants de type II.

\begin{tabular}{|c|c|c|c|}
\hline Matériau & $\begin{array}{c}\text { Supraconductivité } \\
\text { découverte en }\end{array}$ & $\boldsymbol{T}_{\mathbf{c}}(\mathbf{K})$ & $\begin{array}{c}\boldsymbol{H}_{\mathbf{c 2}} \text { (teslas) } \\
\text { (à } \mathbf{T}=\mathbf{0})\end{array}$ \\
\hline $\mathrm{Nb}$ & 1931 & 9,2 & 0,4 \\
\hline $\mathrm{Pb}_{0,7} \mathrm{In}_{0,3}$ & 1932 & 6 & 0,4 \\
\hline $\mathrm{NbTi}$ & 1941 & 9,2 & 15 \\
\hline $\mathrm{Nb}_{3} \mathrm{Sn}$ & 1954 & 18,3 & 30 \\
\hline $\mathrm{Nb}_{3} \mathrm{Ge}$ & 1971 & 23,1 & 37 \\
$\mathrm{YBa}_{2} \mathrm{Cu}_{3} \mathrm{O}_{7}$ & 1987 & 92 & $>100$ \\
\hline $\mathrm{MgB}_{2}$ & 2001 & 42 & 55 \\
\hline $\mathrm{SmFeAsO}$ & 2008 & 55 & $>90$ \\
\hline
\end{tabular}
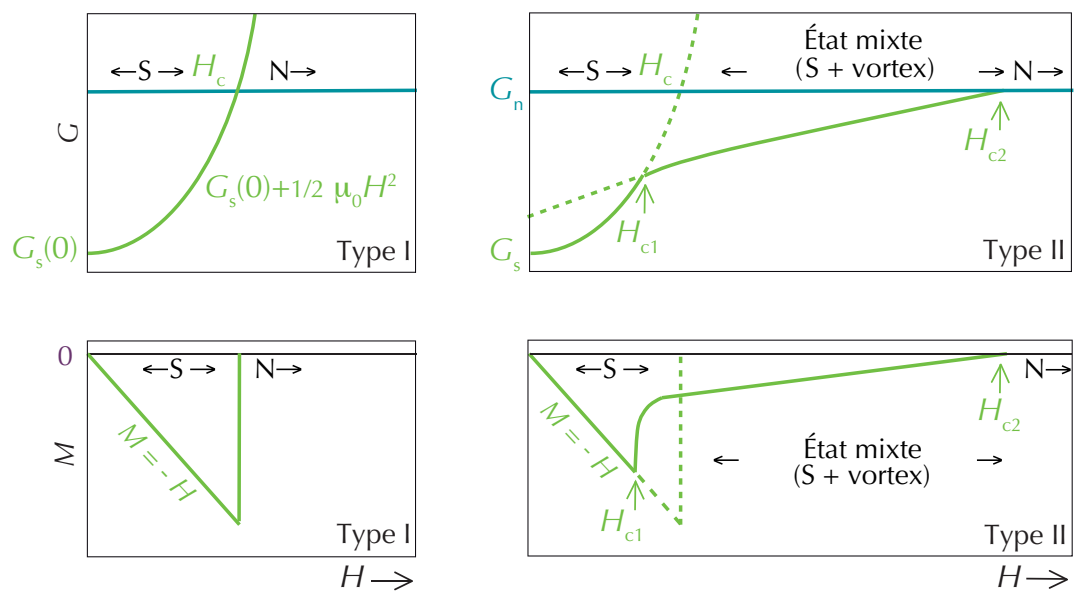

2. Dépendance en champ magnétique $H$ de l'énergie libre $G$ et de l'aimantation $M=-\left(1 / \mu_{0}\right) \mathrm{d} G / \mathrm{d} B$, pour des supraconducteurs de type I et de type II $\left(\mu_{0}=4 \pi \times 10^{-7} \mathrm{H} \cdot \mathrm{m}^{-1}\right)$. Dans l'état normal, $G=G_{\mathrm{n}}$ est constant (ligne bleue), tandis que dans l'état supraconducteur $G$ croît en fonction de $H$ (en vert). Dans les supraconducteurs de type l, il n'y a que deux phases thermodynamiques : l'état supraconducteur (S) pour lequel le flux magnétique est complètement expulsé $(M=-H, B=0)$, et l'état normal $(N)$ pour lequel $M=0$. Le matériau retourne à l'état normal par une transition du premier ordre au champ critique thermodynamique $H_{\text {c. Pour un }}$ supraconducteur de type II, l'expulsion n'est complète que si $H$ est inférieur au premier champ critique $H_{c 1}$. Au-delà, le matériau supraconducteur admet des « vortex ». Comme il contient alors des zones à l'état normal, on parle d' "état mixte ". l'énergie libre de l'état mixte croise celle de l'état normal à $H=H_{c 2}$. 


\section{Exclusion du flux magnétique et effet Meissner} Encadré 1

L'effet Meissner désigne l'expulsion totale du champ magnétique (indiqué par les lignes sur la figure a) du volume d'un matériau supraconducteur, et ceci indépendamment de la manière dont le champ a été appliqué. C'est une des conséquences de la nature quantique macroscopique de l'état supraconducteur. Une description semi-classique d'un métal hypothétique de résistance nulle aboutit à l'exclusion du champ magnétique, seulement si celui-ci est appliqué après un refroidissement en dessous de $T_{\mathrm{C}}$; si on applique le champ avant de refroidir, elle prédit que le flux magnétique reste piégé. La théorie quantique de la supraconductivité prévoit l'expulsion du champ électromagnétique dans tous les cas.

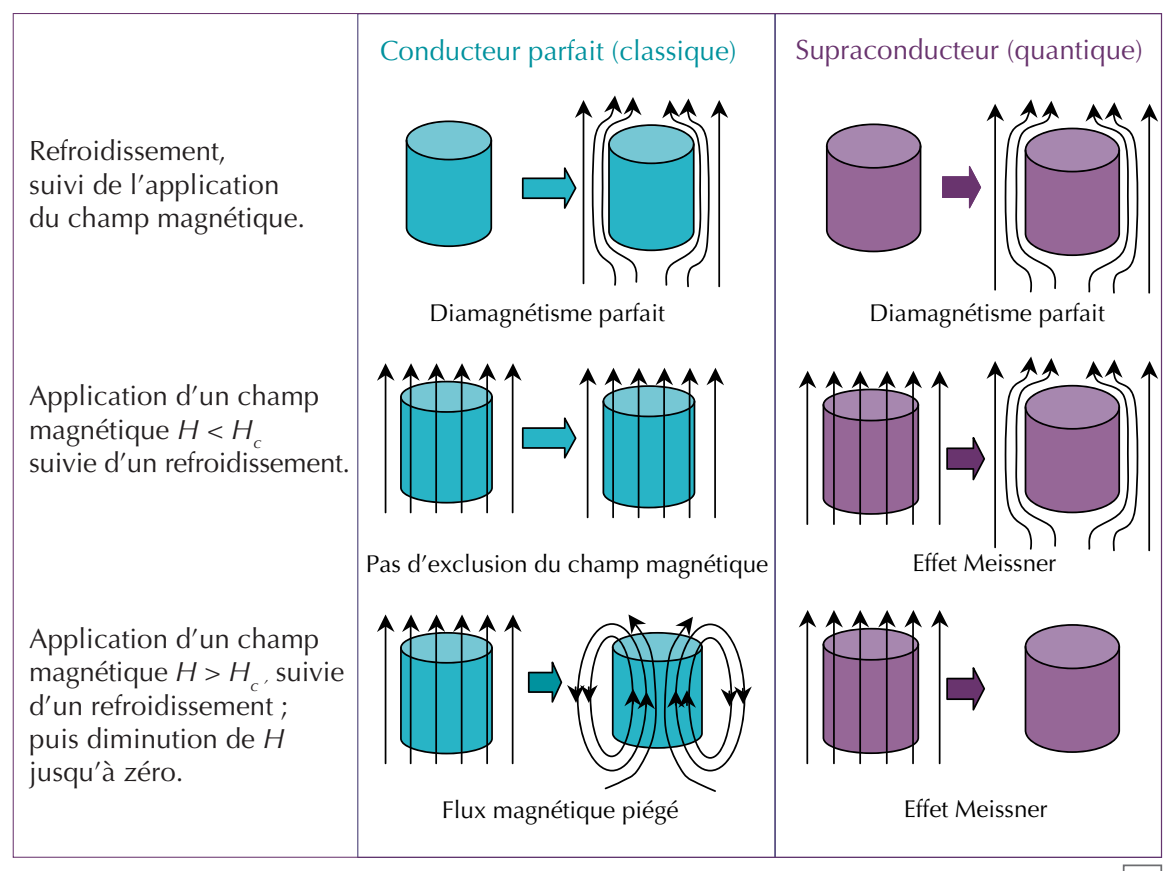

La théorie quantique de la supraconductivité aboutit notamment aux équations de London, $\lambda^{2} \nabla^{2} \mathbf{B}=\mathbf{B}, \lambda^{2} \nabla^{2} \mathbf{E}=\mathbf{E}$ (B est l'induction magnétique, c'est-à-dire la densité de flux magnétique, E est le champ électrique). Lorsque combinées avec les équations de Maxwell, celles-ci montrent comment l'exclusion du flux magnétique est assurée par la génération d'un courant de surface non dissipatif (lignes vertes sur la figure b), créant au sein du supraconducteur un champ magnétique égal mais opposé au champ appliqué. La figure b

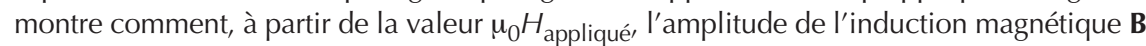
décroît exponentiellement en fonction de la distance à la surface. La longueur caractéristique de la décroissance est la longueur de pénétration de London, $\lambda$.

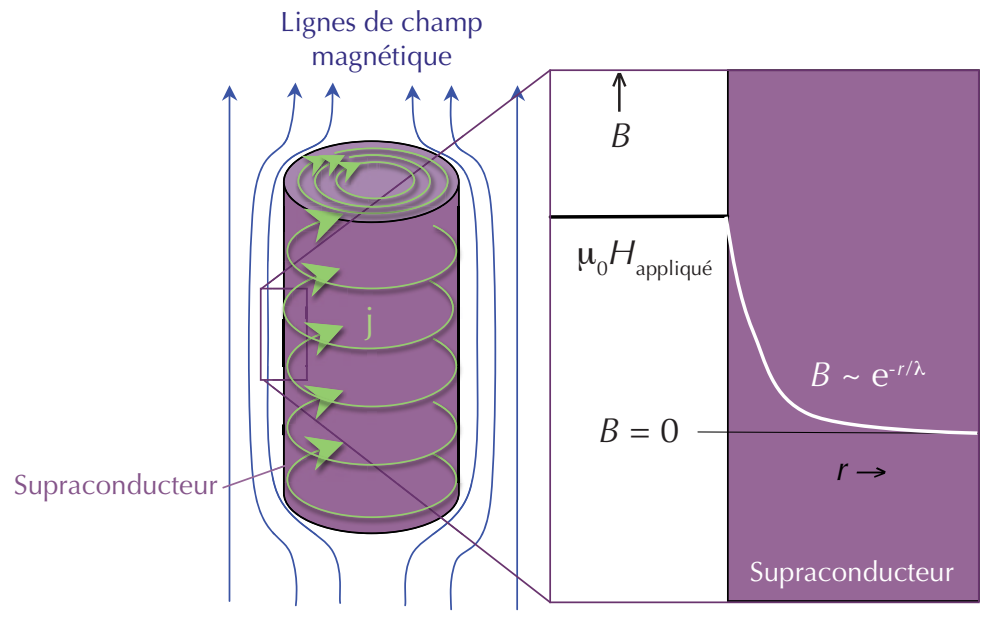

$\gg>$

aujourd'hui largement dépassées par celles des oxydes à haute température critique [6], avec des $T_{c}$ pouvant atteindre $135 \mathrm{~K}$ et des $H_{\mathrm{c} 2}(\mathrm{a} T=0)$ bien au-delà de 100 teslas (voir tableau). Toutefois, leurs meilleures propriétés métallurgiques (bonnes ductilité et résistance à la rupture) font que les alliages de niobium sont toujours à la base de la majorité des applications de la supraconductivité, comme les aimants utilisés dans l'Imagerie à Résonance Magnétique, indispensable dans la médecine moderne, et dans le Large Hadron Collider du CERN. Du fait de son premier champ critique $H_{\mathrm{c} 1}$ exceptionnellement élevé ( $\left.140 \mathrm{mT}\right)$, le niobium est utilisé pour la fabrication de cavités résonnantes dans les accélérateurs de particules; en effet, ces cavités doivent être utilisées dans l'état Meissner $\left(H<H_{\mathrm{c} 1}\right)$ et non pas dans l'état mixte, car la présence de vortex mis en oscillation par le champ radiofréquence conduirait à un réchauffement catastrophique.

\section{Gorter, Casimir et les frères London}

La compréhension de la supraconductivité a dû attendre de nombreuses années. Cornelis Gorter et Hendrik Casimir ont, en 1932, démontré la relation thermodynamique liant la discontinuité de la chaleur spécifique et la dérivée en température $\mathrm{d} H_{\mathrm{c}}(T) / \mathrm{d} T$ à $T=T_{\mathrm{c}}$. Ceci a permis de comprendre les expériences conduites en Allemagne par Walter Meissner, qui a montré en 1933 que le flux magnétique était totalement expulsé du volume du supraconducteur, un effet connu aujourd'hui comme l'effet Meissner.

En 1935, les frères Heinz et Fritz London postulèrent les équations fondamentales gouvernant le comportement électromagnétique des supraconducteurs. Elles décrivent notamment comment, en pénétrant une distance $r$ au sein du matériau supraconducteur, l'induction magnétique $B$ décroît exponentiellement en $r / \lambda$, avant de s'annuler (encadré 1).

La longueur de pénétration de London, $\lambda=\left[m /\left(n_{\mathrm{s}} e^{2} \mu_{0}\right)\right]^{1 / 2}$, dépend du rapport entre la densité des électrons supraconducteurs $n_{\mathrm{s}}$ et la masse effective électronique $m$. Elle est donc caractéristique du matériau, et vaut généralement, à basse température, de l'ordre de 50 à $200 \mathrm{~nm}$. La dépendance en température de $\lambda$ a été expliquée par Gorter et Casimir en termes de la coexistence de deux fluides électro- 
niques, le "superfluide " de densité $n_{\mathrm{s}}$ et un fluide d'électrons "normaux " de densité $n-n_{\mathrm{s}}$ ( $n$ étant la densité totale d'électrons). Comme $n_{\mathrm{s}}$ chute à zéro à $T_{\mathrm{c}}, \lambda$ diverge pour $T \rightarrow T_{c}$ (fig. 1c), entraînant la réadmission du champ.

Les équations de London ne peuvent être déduites à partir d'une approche purement classique, car celle-ci conduit à une valeur constante mais arbitraire de $B$ au sein du supraconducteur, tandis qu'en réalité $B=0$. Dès 1935, les frères London comprirent que l'effet Meissner traduit le caractère quantique de la supraconductivité.

\section{La théorie de Ginzburg et Landau}

Ce caractère quantique de la supraconductivité est l'essence de la théorie phénoménologique établie en 1951 par Vitalii Ginzburg et Lev Landau (GL). La théorie GL, valable à proximité de $T_{c}$, est l'extension aux fluides chargés de la théorie de Landau des transitions de phases. GL ont introduit une fonction d'onde complexe $\psi=|\psi| \mathrm{e}^{\mathrm{i} \varphi}$, ou paramètre d'ordre, dont le carré de la norme $|\psi|^{2}$ représente $n_{\mathrm{s}}$ (fig. $1 \mathrm{c}$ ). Ils ont démontré que la variation spatiale de la densité superfluide $n_{\mathrm{s}}$ a lieu graduellement, sur des distances de l'ordre d'une deuxième longueur fondamentale $\xi$, nommée longueur de cohérence. Comme $\lambda, \xi$ est caractéristique du matériau, et dépend tout aussi sensiblement de son degré de pureté.

Le rapport $\kappa \equiv \lambda / \xi$ distingue les supraconducteurs de type I et de type II (fig. 3). Si $\kappa<1 / \sqrt{ } 2$, l'énergie d'une interface entre phase normale et phase supraconductrice dans le matériau est positive. Ce type d'interface ne peut alors se créer spontanément dans le supraconducteur, le matériau est de type I (avec, typiquement, $\xi$ de l'ordre de 30 à $100 \mathrm{~nm}$ ). Dans le cas des supraconducteurs de type II (avec $\xi \sim 1-20 \mathrm{~nm}), \kappa>1 / \sqrt{ } 2$, l'énergie d'interface est négative, et la multiplication de zones normales au sein du supraconducteur est possible (sous la forme de vortex).

\section{La théorie BCS [4]}

En 1950, on découvre qu'en remplaçant un élément constituant le matériau supraconducteur par un isotope plus lourd, $T_{\mathrm{c}}$ diminue. Cet effet isotopique révèle que les vibrations du réseau cristallin (les phonons) jouent un rôle dans le mécanisme à l'origine de la supraconductivité ; ceci a conduit
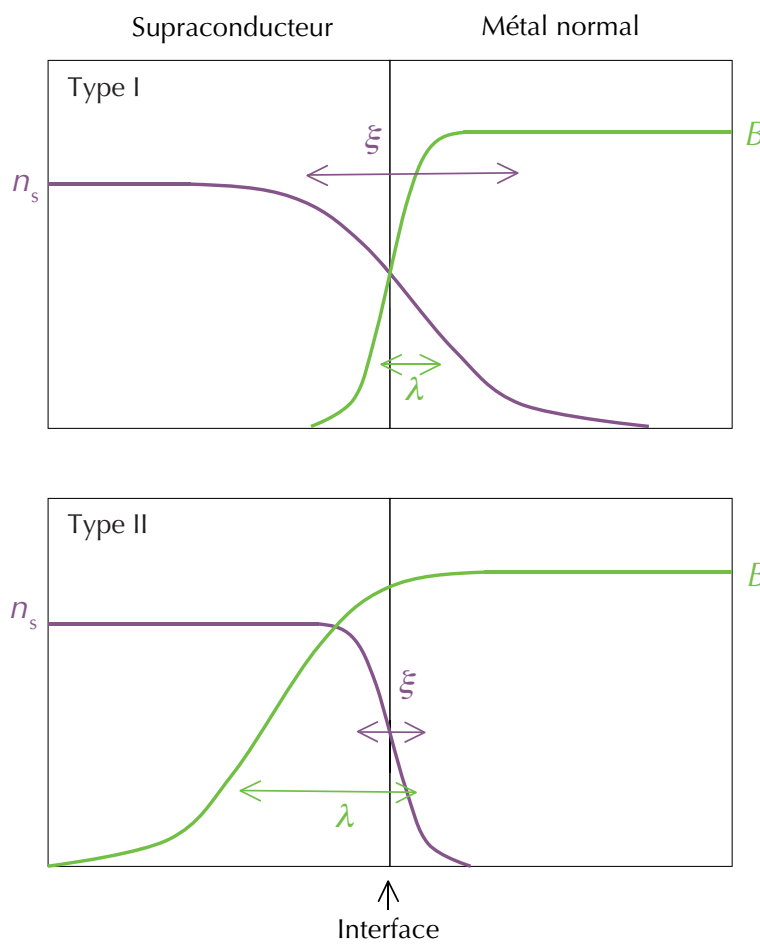

3. Comportement de la densité d'électrons supraconducteurs, $n_{\boldsymbol{s}}$, et de l'induction magnétique $B$, aux abords d'une interface métal normal-supraconducteur, pour les cas ou ce dernier est de type I ou de type II. Pour le supraconducteur de type I, $\xi>\sqrt{2} \lambda$ : la supraconductivité est maintenue dans une large couche superficielle d'épaisseur $\xi$, d'où le flux magnétique est expulsé, une situation défavorable énergétiquement. Si le supraconducteur est de type II, $\xi<\sqrt{2} \lambda$ : la densité $n_{\varsigma}$ diminue rapidement aux abords de l'interface, tandis que l'induction magnétique est admise dans une couche relativement large, une situation qui est favorable énergétiquement.

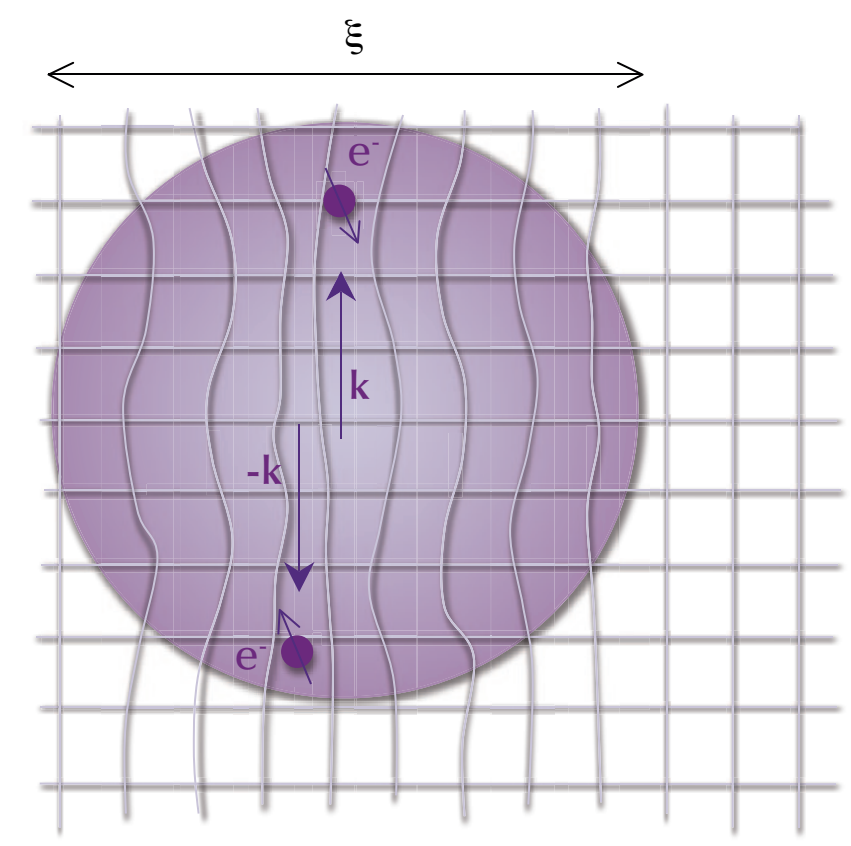

4. Interaction attractive entre électrons, par l'intermédiaire des phonons. Dans un solide conducteur, le passage d'un électron (violet, avec charge -e) polarise temporairement les ions (de charge positive), en les attirant sur son passage. Pour les ions, la durée de cette perturbation est très courte, de l'ordre de $10^{-18} \mathrm{~s}$. Les ions vont se mettre en mouvement avec une période typique de vibration du réseau, de l'ordre de $10^{-12} \mathrm{~s}$, mouvement qui continuera bien après que la perturbation électronique ait disparu. La vibration des ions jouera alors le rôle d'un potentiel attractif pour un autre électron : on dit que l'on a une interaction électron-électron retardée, portée par les vibrations ioniques (phonons). L'interaction attractive est limitée aux électrons situés dans une bande d'énergie $\hbar \omega_{D}$ autour du niveau de Fermi (voir encadré 2). Dans la théorie BCS, l'énergie totale du système d'électrons supraconducteurs est minimale pour des paires d'électrons d'impulsions et de spins opposés, ( $\mathbf{k} \uparrow$ et $-\mathbf{k} \downarrow)$. Cela donne lieu à des paires de Cooper (d'étendue spatiale $\sim \xi$ ), dont l'état est un singulet de spin. 


\section{Bandes interdites et spectroscopies}

Encadré 2

Les électrons étant des fermions, les états électroniques d'un métal ne peuvent être occupés que par un seul électron. L'électron ayant un spin 1/2, il y a deux états de spin possibles pour chaque état orbital, de vecteur d'onde $\mathbf{k}=\left(k_{\mathrm{x}}, k_{\mathrm{y}}, k_{\mathrm{z}}\right)$. Les états sont occupés un à un par les électrons du système, en partant des états de plus faible énergie $\varepsilon$ (fig. a). L'occupation $n(\varepsilon)$ en fonction de $\varepsilon$ a une coupure nette à l'énergie $\varepsilon_{\mathrm{F}}$ du dernier niveau occupé, le niveau de Fermi.
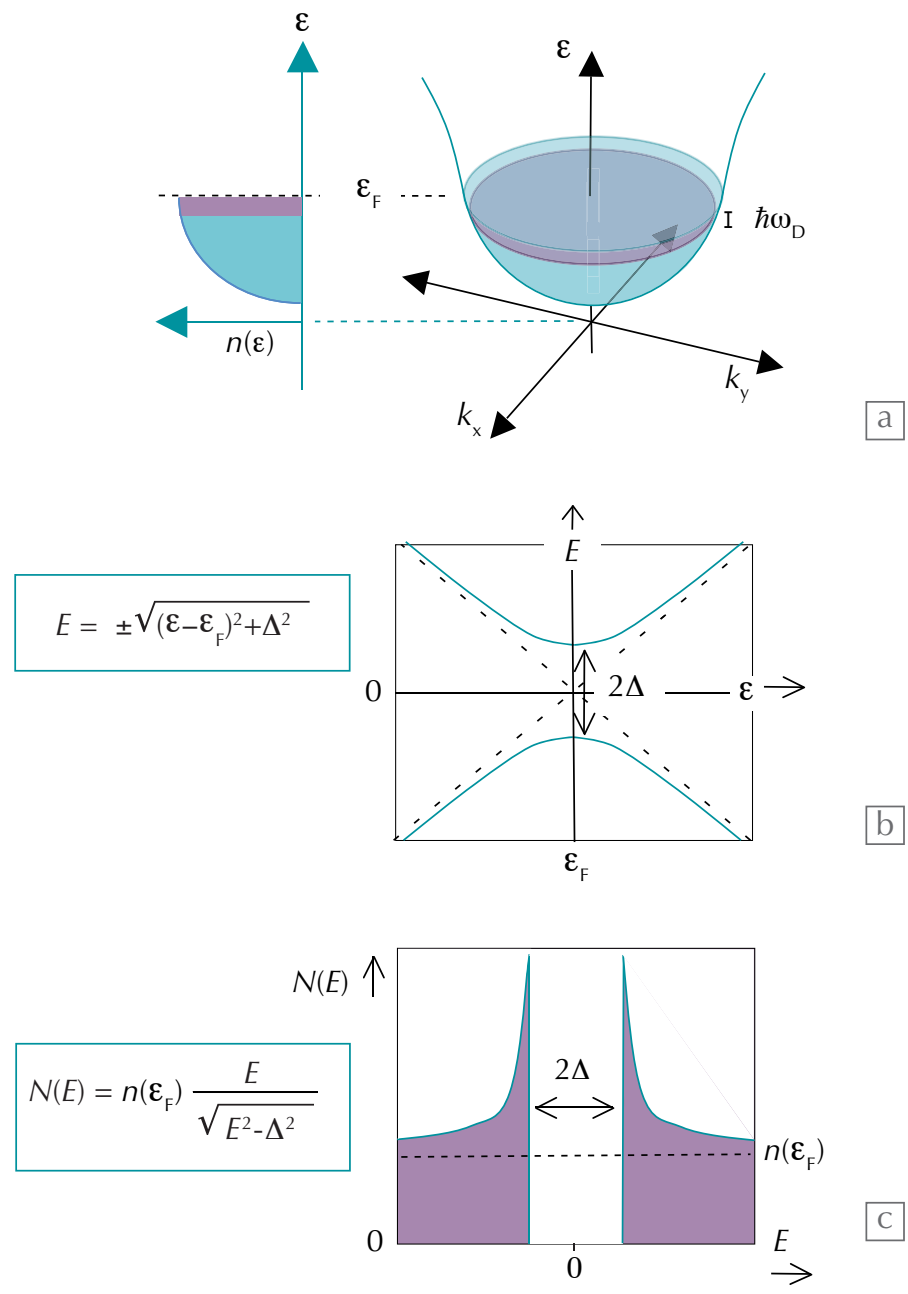

Dans la théorie BCS, seuls les électrons dont l'énergie $\varepsilon$ est comprise dans une étroite bande de largeur $\hbar \omega_{\mathrm{D}}$ autour de $\varepsilon_{\mathrm{F}}$ participent à la supraconductivité. La quantité $\hbar \omega_{\mathrm{D}}$, appelée énergie de Debye, est l'énergie typique des vibrations des ions. Lors du passage à I'état supraconducteur, les états occupés par ces électrons disparaissent, et sont remplacés par des états à deux particules, les paires de Cooper. Cette disparition d'états à une particule peut être sondée, par exemple, par la photoémission résolue en angle (ARPES) [6].

Les paires de Cooper peuvent être dissociées, moyennant une énergie d'activation $E$ qui dépend de l'éloignement entre $\varepsilon$ et l'énergie de Fermi $\varepsilon_{\mathrm{F}}$ (fig. b). La dissociation des paires de Cooper provenant d'électrons avec $\varepsilon=\varepsilon_{\mathrm{F}}$ coûte une énergie $2 \Delta$, où $\Delta$ est la bande interdite du supraconducteur. La densité d'états excités $N(E)$ (fig. c), c'est-à-dire la dérivée $\mathrm{d} \varepsilon / \mathrm{d} E$ de la courbe de la figure b multipliée par la densité d'états du métal normal, est accessible expérimentalement en effectuant une mesure du courant électrique I qui peut passer, grâce à l'effet tunnel quantique, entre un métal normal et le supraconducteur, à une tension $V$ donnée. Si $V$ est inférieur à $\Delta / e$, aucun courant ne passe du métal normal au supraconducteur, car il n'y a pas d'états disponibles dans ce dernier pour accueillir les porteurs de charge. Pour des valeurs plus grandes de $V$, les électrons peuvent passer du métal normal au supraconducteur, dans lequel ils occupent les états correspondant à des paires de Cooper dissociées, ou quasi-particules.
\>

à la théorie microscopique de John Bardeen, Leon Cooper et Robert Schrieffer (BCS) en 1957. L'idée clef de BCS est qu'en dimension 3, la présence d'une interaction attractive $V$ entre électrons dans le matériau, aussi faible soit-elle, rend instable l'état métallique normal. Cette attraction est possible grâce à l'effet de "sur-écrantage " par les phonons de la répulsion coulombienne entre électrons (fig. 4).

La deuxième idée magistrale de BCS est de poser que, dans la phase supraconductrice, on a un nouvel état fondamental, constitué d'une superposition cohérente d'états à deux électrons, appelés paires de Cooper. L'étendue spatiale de ces paires est de l'ordre de $\xi$, bien supérieure aux paramètres de maille du réseau cristallin, tandis que leur nombre est comparable au nombre de mailles. Les paires de Cooper forment ainsi un état quantique macroscopique intriqué ou condensat, avec une valeur unique de la phase $\varphi$ telle que postulée par GL. Cela signifie que l'on ne peut modifier une paire sans modifier l'état de toutes les autres. La diffusion des électrons entre états à phase aléatoire étant à la base de la résistance électrique, une phase $\varphi$ unique implique $R=0$ ! En 1959, Yoichiro Nambu démontre explicitement que la symétrie brisée lors de la transition vers l'état supraconducteur correspond au choix unique de $\varphi$, entre un nombre infini de possibilités. On parle de brisure de symétrie de jauge.

Les états électroniques excités à partir de l'état fondamental BCS, dénommés quasiparticules (encadré 2), sont obtenus en occupant un état de Cooper par un seul électron. L'énergie seuil pour ces excitations est le gap (ou bande interdite) $\Delta(T)$. L'existence de ce gap, déjà proposée par les frères London en 1935, permet de comprendre la variation en $\exp (-\Delta / T)$ de la chaleur spécifique à basse température (fig. 1a). Dans la théorie BCS, la valeur du gap dépend de manière auto-cohérente du nombre de quasi-particules. $T_{\mathrm{c}}$ est définie comme la température à laquelle la densité de quasi-particules devient trop importante pour permettre un condensat stable.

La théorie BCS a permis de prédire très rapidement, et avec grande précision, toutes les propriétés thermodynamiques, électromagnétiques et spectroscopiques des supraconducteurs connus à l'époque, ce qui en fait l'un des succès majeurs de la physique de la matière condensée. 


\section{Quantification du flux et effet Josephson}

C'est par l'unicité de la phase $\varphi$ que se manifeste pleinement la nature quantique de la supraconductivité. Elle est responsable de l'effet de Little et Parks, dans lequel la température critique d'un anneau supraconducteur varie périodiquement en fonction du flux magnétique le traversant. Elle est aussi à la base de la quantification du flux magnétique $\Phi$ traversant une ouverture quelconque créée dans un matériau supraconducteur : $\Phi$ ne peut être qu'un multiple entier du quantum de flux $\Phi_{0}$, dont la valeur $h / 2 e=2,07 \times 10^{-15} \mathrm{~Wb}$ montre directement que l'état fondamental est une superposition d'états à deux particules, avec une charge $2 e$.

Une autre manifestation du caractère quantique à grande échelle de la supraconductivité est l'effet Josephson (encadré 3), où un courant d'électrons appariés peut traverser par effet tunnel une mince barrière isolante, même en l'absence de tension appliquée. L'effet Josephson a de nombreuses applications, en particulier le SQUID, qui permet la mesure de très faibles champs magnétiques (encadré 3). Les SQUIDs sont aujourd'hui utilisés pour mesurer les signaux provenant du cœur ou du cerveau humain. Ils permettent également de nombreuses expériences en mécanique quantique, comme celles liées à l'intrication quantique à l'échelle macroscopique dans des dispositifs solides.

\section{Pour conclure}

La supraconductivité, découverte il y a 100 ans, nous ouvre les portes du monde quantique, mais se rend également fort utile dans de nombreux domaines, que ce soit en recherche fondamentale comme dans la vie de tous les jours. Le nombre de matériaux supraconducteurs est aujourd'hui incroyablement varié : la supraconductivité se manifeste non seulement dans la majorité des éléments simples (voir figure p. 4) et de nombreux alliages, mais aussi dans des sels organiques, des chalcogénures, des oxydes de cuivre [6], des pnictures, et même des semi-conducteurs dopés [7]. Il est fort probable que nous ne sommes pas à notre dernière surprise dans ce domaine passionnant. I

\section{Effet Josephson}

La conductivité électrique à travers une très fine barrière isolante séparant deux métaux est petite mais pas nulle, car les électrons peuvent la franchir par effet tunnel quantique. Le même effet existe pour deux supraconducteurs (fig. a). Or, la cohérence des paires de Cooper, qui se manifeste par la valeur bien définie de la différence $\varphi_{1}-\varphi_{2}$ des phases des états fondamentaux de part et d'autre de la barrière, apporte une richesse supplémentaire. Comme prédit en 1962 par Brian Josephson, le courant tunnel est une fonction périodique de cette différence de phase. Le plus souvent, $I=I_{0} \sin \left(\varphi_{1}-\varphi_{2}\right)$, où $I_{0}$ est déterminé par les détails de la jonction et par la valeur du gap supraconducteur $\Delta$.

Cet effet Josephson donne lieu à de nombreux phénomènes fascinants et utiles. Par exemple, un flux magnétique $\Phi$ peut passer par la barrière, mais est écranté au sein des supraconducteurs. Toutefois, sa présence affecte les phases, de manière à ce que $\Phi=\left(\Phi_{0} / \pi\right)\left(\varphi_{1}-\varphi_{2}\right)$ (où $\Phi_{0}=h / 2 e=2 \times 10^{-15} \mathrm{~Wb}$ est le quantum de flux). La différence des phases donne lieu à un courant $I=I_{0} \sin \left|\pi \Phi / \Phi_{0}\right|$ non nul. La jonction Josephson se comporte donc comme une inductance non linéaire.

La variation temporelle de $\Phi$ provoque l'apparition d'une tension $V$. Or, le flux est lié à la différence des phases. La tension $V$ à travers la jonction est donc associée au changement dans le temps de celle-ci, par la relation $V=\left(\Phi_{0} / 2 \pi\right) \mathrm{d}\left(\varphi_{1}-\varphi_{2}\right) / \mathrm{d} t$. Cette deuxième relation de Josephson implique qu'une tension continue à travers la jonction donne lieu à un courant électrique périodique dans le temps.
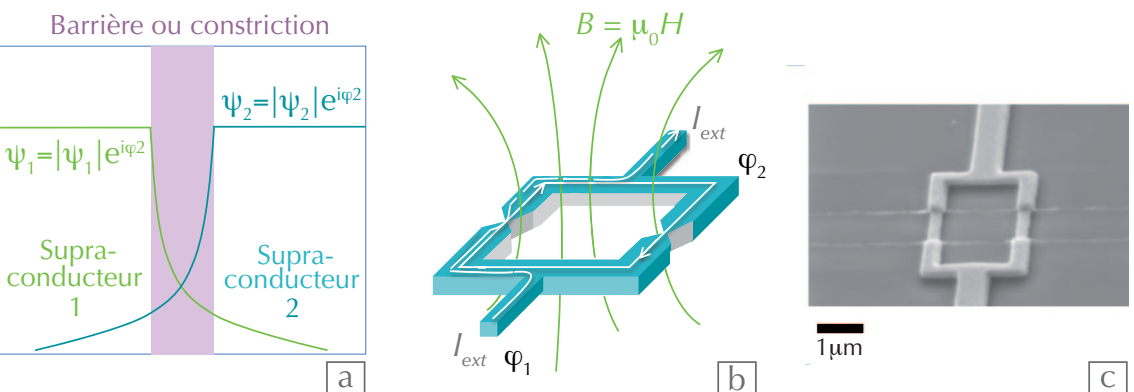

L'un des dispositifs supraconducteurs les plus utilisés, "le dispositif supraconducteur à interférence quantique ", ou SQUID, illustré par le schéma (b) et la photographie (c), consiste en une boucle supraconductrice, interrompue par deux jonctions. Celles-ci peuvent être des jonctions Josephson, mais aussi de simples constrictions. Le SQUID exploite la propriété que le flux $\Phi$ à travers la boucle doit être un multiple entier $(n)$ de $\Phi_{0}$, et que le courant électrique $I$ à travers les jonctions est limité à $I_{0}$. Le courant, indiqué par les lignes blanches sur la boucle supraconductrice bleue dans (b), est la somme du courant injecté de l'extérieur, $I_{\text {ext' }}$ et du courant $I_{\text {ind }}$ induit par le champ magnétique $H$ (lignes vertes). $I_{\text {ind }}$ s'ajustera afin que, toujours, $\Phi=n \Phi_{0}$. Si l'on augmente $H$ à partir de zéro, $l_{\text {ind }}$ croît, jusqu'à ce que $I_{0}$ soit atteint dans I'une des jonctions. Aucun courant externe ne peut alors passer le SQUID. Si I'on augmente $H$ davantage, la jonction en question laisse passer un quantum de flux. On a alors $\Phi=(n+1) \Phi_{0}$. Le signe de $I_{\text {ind }} s^{\prime}$ inverse, et le courant externe que I'on peut passer augmente de nouveau. La valeur maximale de $I_{\text {ext }}$ est donc une fonction périodique de $H$. Sa mesure permet la détection de champs extrêmement faibles, avec la précision de $2 \times 10^{-15} \mathrm{~Wb}$ par unité d'aire de la boucle.

\section{Références}

1• H. Kamerlingh Onnes, Comm. Phys. Lab. Univ. Leiden 120b, 1911 (téléchargeable sur le site www.w2agz.com/SuperWiki.htm).

2• D. van Delft et P.H. Kes, Physics Today, 63 (2010) 38.

3•W.H. Keesom, conférence du 10 avril 1934 devant la Société Française de Physique, J. Phys. Radium 5, 7 (1934) 373 (disponible sur hal.archives-ouvertes.fr).
4• P. Nozières, Reflets de la Physique n7 (2007), p. 14.

$5 \bullet$ C.J. van der Beek et F. Lévy-Bertrand.

$6 \bullet Y$. Sidis et $P$. Bourges.

7• T. Klein, X. Blase, C. Marcenat, C. Chapelier et E. Bustarret.

Les articles des références 5, 6 et 7 sont à paraître dans la suite de ce dossier. 